Trauma Berufskrankh 2004 - 6 [Suppl 3] : S351-S356 DOI 10.1007/s10039-003-0722-3

Online publiziert: 30. April 2003

(c) Springer-Verlag 2003
S.A. Esenwein ${ }^{1}$ K.F. Hopf ${ }^{1} \cdot$ M. Dudda ${ }^{1}$ A. Pommer ${ }^{2}$. F. Kutscha-Lissberg ${ }^{1} \cdot$ G. Muhr ${ }^{1}$ ${ }^{1}$ Chirurgische Klinik mit Poliklinik, BG Kliniken Bergmannsheil, Ruhr-Universität Bochum

${ }^{2}$ Klinik für Unfall- und Wiederherstellungschirurgie, Helios Klinikum Wuppertal, Universität Witten-Herdecke

\title{
Niederenergetischer, gepulster Ultraschall zur Beeinflussung der Knochenregeneration
}

\section{Klinische Anwendung und wissenschaftliche Grundlagen}

Klinisch wurde die adjuvante Ultraschallbehandlung frischer und verzögert heilender Knochenbrüche bereits frühzeitig angewandt. Schon 1990 berichtete Knoch [10] über 20-jährige Erfahrungen an 2500 mit Ultraschall behandelten Patienten. In allen Fällen wurde mit einem relativ hoch dosierten und kontinuierlichen Ultraschallsignal (Frequenz $800 \mathrm{kHz}$, Intensität $0,1-0,5 \mathrm{~W} / \mathrm{cm}^{2}$ ) gearbeitet, welches zu einem Temperaturanstieg im umliegenden Gewebe führt und bei lokaler Applikation von länger als 3 min die Gefahr von Hitzenekrosen mit sich bringt. Um diesen Effekt der Gewebeerwärmung und somit die Gefahr von Hitzenekrosen auch bei längerer Anwendungsdauer zu minimieren, wurde zwischenzeitlich ein System entwickelt, welches ein niederenergetisches, gepulstes Signal verwendet [3].

Niederenergetischer, gepulster Ultraschall

Niederenergetischer, gepulster Ultraschall wird seit 1983 zur Beschleunigung der Frakturheilung eingesetzt. Vorausgegangene tierexperimentelle Studien zeigten, dass die Knochenheilung auch durch dieses Verfahren beschleunigt wird und es zu einer vermehrten Kallusbildung kommt [3]. Das Verfahren besitzt in den Vereinigten Staaten von Amerika seit dem 17.10.1994 die Zulassung der Food and Drug Administration (FDA), nachdem dessen stimulative Eigenschaften bei der Knochenbruchheilung und der Therapie von Pseudarthrosen in 2 prospektiven, doppelblinden und randomisierten Studien am Menschen $[6,11]$ nachgewiesen werden konnten. Bei den beiden zitierten Studien wurde eine tägliche Behandlungszeit von 20 min eingehalten und mit einem niederenergetischen, gepulsten Ultraschallsignal (Frequenz 1,5 MHz, gepulst mit $1 \mathrm{kHz}$, Signallänge $200 \mu \mathrm{s}$, Leistung $117 \mathrm{~mW}$; Intensität $30 \mathrm{~mW} / \mathrm{cm}^{2}$ ) gearbeitet. Die Eigenschaften des angewandten niederenergetischen, gepulsten Ultraschallsignals lagen dabei im Bereich der als sicher geltenden diagnostischen Anwendungen.

Ein kommerzielles Gerät mit entsprechenden physikalischen Parametern wird seit geraumer Zeit von der Industrie (Smith \& Nephew GmbH, Produktbereich gebildeten Knochengewebes wird in der Frakturzone signifikant gesteigert [8]. 

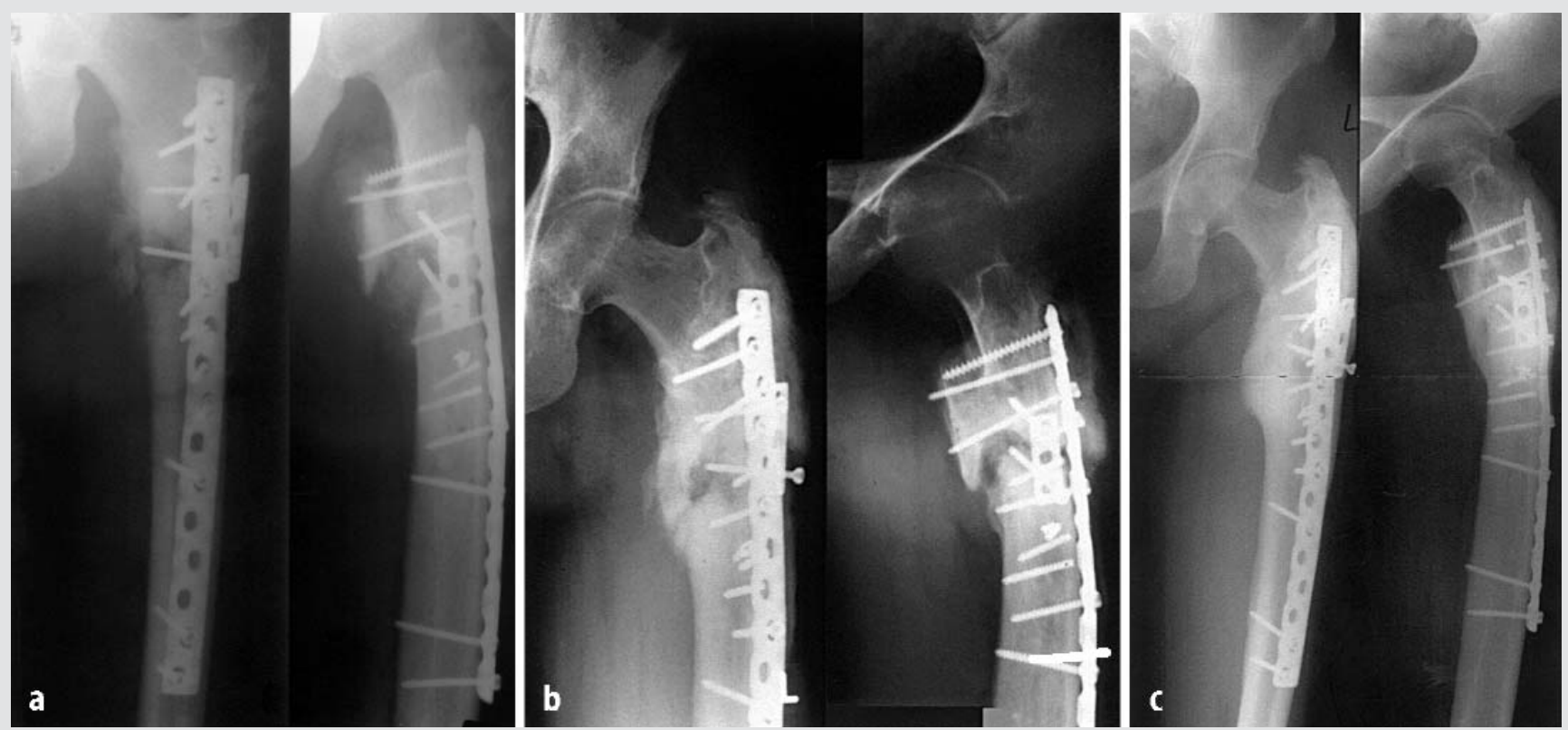

Abb. 1a-c $\Delta$ 36-jähriger männlicher Patient, subtrochantere Femurfraktur mit großer medialseitiger Defektzone, auswärtige primäre osteosynthetische Versorgung mittels Gleitnagel nach Friedl, 15 Tage nach Erstoperation Zuweisung bei bestehender Frakturdehiszenz und vorliegender Außenrotationsstellung von $20^{\circ}$; im Verlauf 3-malige Reosteosynthese mit mehrfacher Spongiosaplastik aufgrund wiederholtem Osteosyntheseversagen notwendig, zuletzt mit Ausriss der zuvor eingebrachten breiten 10-Loch-LCDC-Platte. a Versorgungsergebnis nach letzter Operation. b Ausbildung einer Pseudarthrose 6 Monate nach dem letzten chirurgischen Eingriff. Die Indikation zur Durchführung der Therapie mittels niederenergetischem, gepulstem Ultraschall wird gestellt. c Ausheilungsergebnis nach 4-monatiger Therapie mittels niederenergetischem, gepulstem Ultraschall. Knöcherne Konsolidierung unter schmerzfreier Vollbelastung der betroffenen Extremität ist gegeben

Exogen, 22869 Schenefeld, Deutschland) angeboten. Zur wirksamen Behandlung muss der Schallkopf des Geräts über der Defektzone zu liegen kommen, was vor Behandlungsbeginn unter Durchleuchtung festgelegt und auf der Haut des $\mathrm{Pa}$ tienten markiert wird. Nach Geräteeinweisung führen die Patienten dann die Behandlung selbstständig zu Hause durch.

Im Folgenden soll ein Überblick über die klinische Anwendung von niederenergetischem, gepulstem Ultraschall bei Störungen der knöchernen Regeneration und über die wissenschaftlichen Grundlagen zum Wirkmechanismus des Verfahrens gegeben werden. Dabei werden die eigenen Behandlungsergebnisse mitberücksichtigt.

\section{Klinische Anwendungen von niederenergetischem, gepulstem Ultraschall}

Durch die Anwendung von niederenergetischem, gepulstem Ultraschall konnte in klinischen und experimentellen Studien eine Verbesserung der Frakturheilung nachgewiesen werden $[2,3,6,11,13,14$, $15,17]$. Dabei konnte eine Verkürzung der Heilungszeit von bis zu $38 \%$ im Vergleich zu unbehandelten Patienten belegt werden.

\section{Anwendungen \\ bei gestörter Frakturheilung}

In einer prospektiv konsekutiven Anwendungsbeobachtung wiesen Mayr et al. [17] die Wirksamkeit von niedrig intensivem, gepulstem Ultraschall zur Behandlung von Frakturheilungsstörungen nach. 100 Patienten wurden in die Studie eingeschlossen. In 64 Fällen lag ein Frakturalter von über 120 Tagen und in 36 Fällen ein Frakturalter von über 240 Tagen zugrunde. In allen Fällen wurde die Ultraschalltherapie als Alternative zu der ansonsten als indiziert angesehenen operativen Therapie der Frakturheilungsstörung durchgeführt.
Insgesamt konnte bei dem oben genannten Patientenkollektiv mit gestörter Frakturheilung (verzögerte Knochenbruchheilung bzw. Pseudarthrosenbildung) eine Heilungsrate von $86 \%$ unter Anwendung von niederenergetischem, gepulstem Ultraschall erreicht werden. Auch die eigenen klinischen Behandlungsergebnisse an bisher 14 Patienten außerhalb einer Studie zeigten die Wirksamkeit der Ultraschallbehandlung bei der Pseudarthrosentherapie (• Abb. 1).

\section{Anwendungen \\ bei frischen Skaphoidfrakturen}

Bei der Behandlung von frischen Skaphoidfrakturen konnte im Rahmen einer prospektiv randomisierten Untersuchung an 30 Patienten ebenfalls eine Verkürzung der Heilungszeit durch niederenergetischen, gepulsten Ultraschall belegt werden [15]. Die Heilungszeit der Frakturen verkürzte sich von $62 \pm 19,2$ Tagen in der unbehandelten Gruppe auf 43,2 $\pm 10,9$ Tage 
in der Ultraschallgruppe. Die mittels sagittalen computertomographischen Schnittbildaufnahmen ermittelte Durchbauung der Frakturzone zeigte 4, 6 und 8 Wochen nach dem Unfall einen signifikanten Vorteil $(p<0,05)$ der mit Ultraschall behandelten Patienten.

Unterstützung der Regeneratreifung bei der Kallusdistraktion

Eine weitere Herausforderung für den Einsatz von Ultraschall stellt die Unterstützung der Regeneratreifung bei der Kallusdistraktion dar [4, 23]. Im Rahmen einer prospektiven, randomisierten Studie an der Klinik des Erstautors wurden bisher 32 Patienten mit Distraktionskortikotomien $>4 \mathrm{~cm}$ nachuntersucht, um festzustellen, ob sich bei der Kallusdistraktion eine Stimulation der Regeneratbildung und -reifung durch die Anwendung von niederenergetischem, gepulstem Ultraschall erzielen lässt.

Beide Patientengruppen (mit und ohne Anwendung von Ultraschall) waren hinsichtlich ihrer demographischen Daten vergleichbar. Die durchschnittliche Transportstrecke betrug in der Ultraschallgruppe $74 \mathrm{~mm}$ und in der Kontrollgruppe $75 \mathrm{~mm}$. Die Indikation zur Kallusdistraktion ergab sich in 9 Fällen bei primärem Substanzdefekt nach Fraktur der unteren Extremität, bei 23 Patienten wurde eine Segmentresektion zur Therapie einer vorbestehenden Osteitis durchgeführt. 16 von 32 Patienten führten eigenständig nach Geräteeinweisung durch den behandelnden Arzt während 20 min täglich eine Therapie mittels niederenergetischem, gepulstem Ultraschall über der Regeneratzone durch.

Die Qualität des gebildeten Regenerats wurde sonographisch und radiologisch in 2- bis 4-wöchigen Abständen im Rahmen der ambulanten Wiedervorstellung der Patienten beurteilt. Ferner wurde die Kallusdichte in den standardisierten Röntgenaufnahmen mittels digitaler Bildanalyse gemessen. Die statistische Auswertung der ermittelten radiologischen Dichtewerte innerhalb der Regeneratstrecke erfolgte mittels ANOVA-Varianzanalyse. Endpunkt der Studie waren die Transportzeit sowie die Behandlungszeit bis zur Fixateurentfernung.

Trauma Berufskrankh 2004 - 6 [Suppl 3] : S351-S356

DOI 10.1007/s10039-003-0722-3

C) Springer-Verlag 2003

\section{S. A. Esenwein · K. Friedemann Hopf · M. Dudda · A. Pommer · F. Kutscha-Lissberg · G. Muhr}

\section{Niederenergetischer, gepulster Ultraschall zur Beeinflussung der Knochenregeneration. Klinische Anwendung und wissenschaftliche Grundlagen}

Zusammenfassung

Durch die Anwendung von niederenergetischem, gepulstem Ultraschall kann die Knochenheilung beschleunigt werden. Die vorliegende Arbeit soll einen Überblick über die klinischen Anwendungsmöglichkeiten von niederenergetischem, gepulstem Ultraschall bei Störungen der knöchernen Regeneration und über die wissenschaftlichen Grundlagen zum Wirkmechanismus des Verfahrens geben. Dabei werden neben Erfahrungen aus der Literatur auch eigene experimentelle Resultate und klinische Behandlungsergebnisse mitberücksichtigt. Die präsentierten Ergebnisse

\section{Low-intensity, pulsed ultrasound and its influence on bone regeneration. Clinical results and scientific background}

\section{Abstract}

Bone healing can be accelerated by the use of low-intensity, pulsed ultrasound. This article offers an overview of the possibilities for its clinical application as well as its scientific basics as far as its effect mechanism is concerned. Apart from experiences cited in the literature, our own experimental and clinical treatment results are also demonstrated. The results presented show a stimulatory effect of low-intensity, pulsed ultrasound on fracture healing. In our opinion, the belegen den stimulierenden Effekt von niederenergetischem, gepulstem Ultraschall auf die Frakturheilung. Insbesondere in Grenzsituationen wie der Kallusdistraktion und der Therapie von Pseudarthrosen erscheint uns deshalb der klinische Einsatz des Verfahrens als gerechtfertigt.

Schlüsselwörter Gepulster Ultraschall · Kallusdistraktion . Extremitätenverlängerung · Frakturheilungsstörung · Pseudarthrose clinical application of low-intensity, pulsed ultrasound is indicated especially in borderline situations such as callus distraction and the therapy of pseudarthrosis.

Keywords

Pulsed ultrasound - Callus distraction Limb lengthening · Delayed fracture healing . Pseudarthrosis 


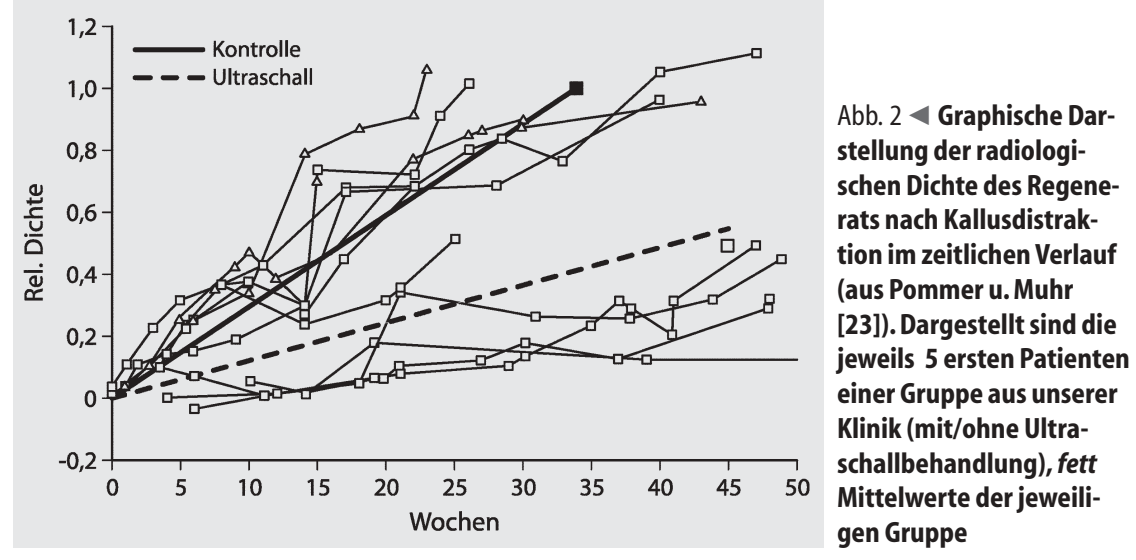

Die mittels digitaler Bildanalyse der standardisierten Röntgenaufnahmen ermittelte radiologische Kallusbildung zeigte im zeitlichen Verlauf eine signifikant raschere Mineralisation (ANOVA, $p<0,01$ ) in der Ultraschallgruppe (• Abb. 2). Die sonographischen Kontrolluntersuchungen erlaubten eine gute Kontrolle in der Frühphase der Kallusbildung, insbesondere waren Störungen der Regeneratbildung rechtzeitig sichtbar. In beiden Gruppen kam es bei jeweils 1 Patienten zum Regeneratversagen, sodass nach Transportabschluss eine Spongiosaplastik mit additiver Plattenosteosynthese erforderlich war. Je 1-mal pro Gruppe wurde eine Unterschenkelamputation vorgenommen, welche in einem Fall aufgrund des Rezidivs einer Osteitis im Dockingbereich mit therapieresistentem Infekt und im anderen Fall aufgrund eines chronischen Weichteildefekts am distalen Unterschenkel notwendig war.

\section{Wissenschaftlicher Hintergrund}

\section{Studienergebnisse}

Der zellbiologische Wirkmechanismus des Ultraschalls auf die Knochenneubildung ist noch nicht vollständig geklärt und wird derzeit in weiterführenden Untersuchungen erforscht. Vorausgegangene Studien [21] konnten die Stimulation der enchondralen Ossifikation von fetalen Mäusemetatarsalia zeigen. In der ultraschallbehandelten Gruppe fanden sich ein signifikant positiver Einfluss auf die Länge der verknöcherten Diaphyse und eine vermehrte Bildung von hypertrophem, teilweise verkalktem Knorpel. Eine mögliche Erklärung für diesen Effekt wäre die Stimulation der Osteoblasten- aktivität und/oder deren Differenzierung, wodurch es zu einer Längenzunahme des verknöcherten diaphysären Anteils kommt. Aufgrund von Genexpressionsanalysen wiesen Yang et al. [30] nach, dass niederenergetischer, gepulster Ultraschall insbesondere die enchondrale Ossifikation stimuliert.

Parvizi et al. [22] zeigten in einer Studie an aus Femurkondylen neugeborener Ratten isolierten Chondrozyten einen Effekt von niederenergetischem, gepulstem Ultraschall. Unter Anwendung des Verfahrens öffneten sich die membranständigen Kalziumkanäle der Zellen. Die Änderung der intrazellulären Kalziumkonzentration war dabei von der Intensität des Ultraschallsignals abhängig. Der Kalziumeinstrom beeinflusste die enzymatische Aktivität der Zellen und führte $\mathrm{zu}$ einer Synthesesteigerung von spezifischen Matrixproteinen [29].

Schmelz [25] stellte in vitro an humanen Fibroblasten- und Osteoblastenpopulationen einen proliferationsfördernden Effekt von niederenergetischem, gepulstem Ultraschall fest. Auch Sun et al. [26] wiesen in vitro einen stimulierenden Effekt der Ultraschallbehandlung auf Knochenzellen nach. An Osteoblasten, die aus Calvarien neugeborener Ratten isoliert wurden, erfolgte ab dem 4. Tag für 20 min täglich die Applikation von niederenergetischem, gepulstem Ultraschall während einer Gesamtversuchsdauer von 10 Tagen. Bei der nach 10 Tagen durchgeführten Zellzahlbestimmung fand sich eine signifikante Zunahme der Osteoblastenpopulation im Vergleich zur Zellzahl in der unbehandelten Kontrollgruppe. Ferner war die alkalische Phosphatase als Leitenzym der Knochenneubildung nach 7-tägiger Ultraschallstimulation im
Kulturmedium signifikant angestiegen [26].

Einzelne, nicht systematische In-vitroStudien an Osteoblastenkulturen zeigten die Expression spezifischer, lokal im Knochen wirkender Wachstumsfaktoren wie IGF-I oder TGF- $\beta[20,24,28]$ nach Stimulation mit niederenergetischem, gepulstem Ultraschall. Dabei vermitteln diese Wachstumsfaktoren ihre Effekte über spezifische Rezeptorenkomplexe [18].

\section{Eigene Untersuchungen}

In eigenen experimentellen Studien an der Abteilung für Chirurgische Forschung der BG Kliniken Bergmannsheil wurden humane osteoblastäre Zelllinien (MG-63 und SAOS-2) hinsichtlich der Synthese osteotropher Zytokine (IL-6, IL-11) unter dem Einfluss von niederenergetischem, gepulstem Ultraschall (Frequenz 1,5 MHz, gepulst mit $1 \mathrm{kHz}$, Signallänge $200 \mu \mathrm{s}$, Leistung $117 \mathrm{~mW}$; Intensität $30 \mathrm{~mW} / \mathrm{cm}^{2}$ ) in vitro untersucht [5]. Anhand einer distinkten Zytokinsynthese konnte die ultraschallvermittelte Zellaktivierung nachgewiesen werden. So wurde in den SAOS-2und MG-63-Kulturen die Freisetzung von IL-6 bzw. IL-11 (spontan und induziert durch bFGF, VEGF, IL-1 $\beta$, TNF- $\alpha$ ) durch die Behandlung mit niederenergetischem, gepulstem Ultraschall unterschiedlich stark moduliert. Während die Synthese von IL-6 aus MG-63 bei Zugabe von FGF durch niederenergetischen, gepulsten Ultraschall positiv beeinflusst wurde, war die IL-11-Synthese aus SAOS-2 unter Zugabe von VEGF und IL-1 $\beta$ erhöht. Diese Ergebnisse weisen auf den stimulierenden Effekt von niederenergetischem, gepulstem Ultraschall auf humane osteoblastäre Zelllinien in vitro hin.

\section{Experimentelle Studien in vivo}

Mayr [13] und Mayr et al. [16] untersuchten an 18 erwachsenen Merinoschafen, inwieweit die tägliche Anwendung von niedrig intensivem Ultraschall für $20 \mathrm{~min}$ zu einer beschleunigten Regeneratreifung nach Kallusdistraktion führt [13, 16]. Hierzu wurde am rechten Metatarsus der Tiere ein Segmenttransport (Latenz: 4 Tage, Distraktionsgeschwindigkeit 1,0 mm/Tag) durchgeführt. Nach Abschluss der 21-tägi- 
gen Distraktionsphase erfolgte während der 63-tägigen Reifungsphase bei der Hälfte der Versuchstiere die Behandlung mittels niedrig intensivem Ultraschall für 20 min täglich. In den zum Zeitpunkt des Versuchsendes am 84. Tag angefertigten hoch auflösenden Röntgenaufnahmen fand sich bei Mayr et al. [16] sowohl für die Reifung als auch für die Menge an gebildetem Kallus ein deutlicher Vorteil zugunsten der Ultraschallgruppe. Computertomographisch ließ sich bei den Tieren der Ultraschallgruppe ein signifikant höherer Mineralgehalt im gesamten Regenerat nachweisen. Die axiale Steifigkeit des neu entstandenen Knochens zeigte mit $6941 \pm 961 \mathrm{~N} / \mathrm{mm}$ vs. $2942 \pm 1913 \mathrm{~N} / \mathrm{mm}$ ebenfalls einen signifikanten Vorteil zugunsten der stimulierten Tiere [13]. Die quantitative histologische Auswertung des am 49. postoperativen Tag fluoreszenzmarkierten Kallusgewebes zeigte bereits zu diesem Zeitpunkt bei den mit Ultraschall behandelten Tieren eine Mineralisation von $65-70 \%$ im Vergleich zu $25-$ $30 \%$ bei den Kontrolltieren, sodass aufgrund der von Mayr [13] und Mayr et al. [16] publizierten tierexperimentellen Ergebnisse auf eine beschleunigte Regeneratreifung nach Kallusdistraktion durch die tägliche Anwendung von niederenergetischem, gepulstem Ultraschall geschlossen werden kann.

Tis et al. [27] untersuchten im Rahmen einer tierexperimentellen Studie an Kaninchen den Einfluss der Therapie mittels niedrig intensivem, gepulstem Ultraschall auf die Regeneratreifung nach Distraktionsosteogenese. Nach Osteotomie der Tibia im mittleren Drittel wurde bei 26 adulten Neuseeländer Kaninchen einseitig ein Fixateur externe von anteromedial an die Tibia angebracht. Nach einer Latenzzeit von 7 Tagen wurde mit jeweils $0,5 \mathrm{~mm}$ alle $12 \mathrm{~h}$ distrahiert. Nach 10 Tagen wurde die Distraktion abgeschlossen und bei 13 Tieren mit der Therapie mit niederenergetischem, gepulstem Ultraschall begonnen. Über eine Zeitdauer von 20 Tagen wurde 1-mal täglich für 20 min mit niederenergetischem, gepulstem Ultraschall behandelt. Nach Abschluss des Versuchs am 37. postoperativen Tag wurden die Regenerate ausgewertet. Radiologisch fand sich in der Behandlungsgruppe 1, 2 und 3 Wochen nach Distraktionsende eine signifikant vermehrte Kallusbildung im Vergleich zur Kontrollgruppe $(p<0,01$; $p<0,008$ bzw. $p<0,05)$. Histomorphometrisch konnten die Autoren [27] signifikant weniger bindegewebige Anteile und vermehrt Knochen in den Regeneraten der Ultraschallgruppe im Vergleich zu denjenigen der unbehandelten Tiere nachweisen $(p<0,05)$.

Nach Machen et al. [12] ist für eine adäquate Regeneratqualität unter dem Einfluss von niederenergetischem, gepulstem Ultraschall eine relativ rigide Montage des zur Kallusdistraktion angewandten Fixateursystems erforderlich. Die genannten Autoren konnten tierexperimentell an 20 adulten Neuseeländer Kaninchen zeigen, dass es bei einem entsprechend gewählten Fixateursystem und daraus resultierender wenig rigider biomechanischer Situation durch die Stimulation mit niederenergetischem, gepulstem Ultraschall zu einer vermehrten Knorpelbildung und $\mathrm{zu}$ einer biomechanisch unterlegenen Kallusbildung im Vergleich zur unbehandelten Kontrolle kommt.

\section{Resümee bisheriger Ergebnisse}

Zusammenfassend lassen die bisherigen experimentellen Studien erkennen, dass es auch zukünftig notwendig sein wird, die Mechanismen der Knochenneubildung unter dem Einfluss von niederenergetischem, gepulstem Ultraschall weiter zu erforschen, um die klinische Anwendung des Verfahrens in Zukunft noch effektiver gestalten zu können. Aus diesem Grund wird der Forschung auf dem Gebiet der Mechanotransduktion an den an der Knochenregeneration beteiligten Zellpopulationen weiterhin eine wesentliche Bedeutung zukommen.

\section{Diskussion}

Unter Berücksichtigung der eigenen positiven Erfahrungen mit niederenergetischem, gepulstem Ultraschall und der berichteten Ergebnisse in der wissenschaftlichen Literatur halten wir die vorgestellte Therapieform für eine viel versprechende Alternative bzw. für ein sinnvolles Adjuvans zur Behandlung von Frakturheilungsstörungen und zur Verkürzung der Regeneratreifung bei der Kallusdistraktion.
Es bleibt zu hoffen, dass künftig der Stellenwert der Therapie mit niederenergetischem, gepulstem Ultraschall auch auf breiter Basis erkannt wird. Derzeit besteht bei der gesetzlichen Krankenversicherung (GKV) im Regelfall eine ablehnende Haltung im Hinblick auf die Übernahme der Therapiekosten. Durch den Bundesausschuss der Ärzte und Krankenkassen wurde mit Wirkung vom 22.3.200o beschlossen, die Therapie mit niederenergetischem, gepulstem Ultraschall in die Richtlinien über die Bewertung ärztlicher Untersuchungs- und Behandlungsmethoden gemäß $\$ 135$ Abs. 1 SGB V (BUB-Richtlinien) als Methode aufzunehmen, die nicht als vertragsärztliche Leistung zu Lasten der Krankenkassen erbracht werden darf (Anlage B der BUB-Richtlinien in der Fassung vom 10.12.1999). Begründet wurde die Vorgehensweise des Bundesausschusses der Ärzte und Krankenkassen zum damaligen Zeitpunkt mit der Auffassung, dass keine ausreichenden, wissenschaftlich belegten Erkenntnisse über die medizinische Wirksamkeit und den Nutzen der Methode vorliegen würden. Die Ergebnisse der beiden prospektiven, doppelblinden Studien [6,11], welche die stimulativen Eigenschaften von niedrigdosiertem, gepulstem Ultraschall bei der Knochenbruchheilung und der Therapie von Pseudarthrosen am Menschen belegen konnten und die in den Vereinigten Staaten von Amerika zur Zulassung des Verfahrens durch die FDA führten, wurden dabei nicht als hinreichend beweiskräftig erachtet oder nicht zur Kenntnis genommen.

Um den Forderungen der gesetzlichen Krankenkassen in Bezug auf die zukünftige Übernahme der Therapiekosten mit niederenergetischem, gepulstem Ultraschall gerecht zu werden, wird derzeit in Deutschland eine randomisierte, kontrollierte, doppelblinde Multizenterstudie zur Wirksamkeit und Verträglichkeit von niederenergetischem, gepulstem Ultraschall bei verzögerter Frakturheilung am Unterschenkel durchgeführt. Eingeschlossen in die Studie werden volljährige Patienten mit einer verzögerten Frakturheilung der Tibia im mittleren oder distalen Schaftdrittel, die mit Marknagel oder Plattenosteosynthese versorgt wurden und mindestens 4 Monate nach der Fraktur 
bzw. dem letzten chirurgischen Eingriff keine komplette Durchbauung der Frakturzone, eine verminderte Kallusbildung bzw. typische Zeichen einer hypertrophen, athrophen oder hypothrophen Pseudarthrose aufweisen. Für Patienten, welche die geschilderten Einschlusskriterien erfüllen, besteht zurzeit noch die Möglichkeit zur Teilnahme an dieser Studie.

Die Frage nach der Notwendigkeit einer solchen klinischen Doppelblindstudie bleibt aber nicht unumstritten, gerade dann, wenn experimentelle Befunde deutliche Unterschiede zwischen der Behandlungs- und der Kontrollgruppe erkennen lassen.

Bei der Kallusdistraktion ist die Durchführung einer Doppelblindstudie mit 2 vergleichbaren Gruppen (randomisiert und prospektiv) selbst im Rahmen von Multizenterstudien nahezu unmöglich, da durch die eingeschränkte Fallzahl, die unterschiedlichen Verletzungsmuster, die differierenden Ausgangsbefunde und das unterschiedliche Alter der behandelten Patienten nur schwer direkt vergleichbare Ergebnisse zu erwarten sind.

\section{Hinweise für die Praxis}

Zusammenfassend halten wir unter Berücksichtigung der eigenen positiven Erfahrungen mit niederenergetischem, gepulstem Ultraschall und der berichteten Ergebnisse in der wissenschaftlichen Literatur die vorgestellte Therapieform für eine mögliche, viel versprechende Alternative bzw. für ein sinnvolles Adjuvans zur Behandlung von Frakturheilungsstörungen und zur Verkürzung der Regeneratreifung bei der Kallusdistraktion.

Bei der breiten oder generellen Anwendung erscheint trotz der positiven Einschätzung zur Wirksamkeit des Verfahrens aber Skepsis geboten, weil ökonomische Aspekte bei der Therapieplanung ebenso in Betracht gezogen werden müssen, wie die mögliche Belastung des behandelten Patienten. Im Fall einer unzureichenden Wirksamkeit kann es sonst zu Verzögerungen bei der Einleitung einer notwendigen operativen Behandlung und zu Schwierigkeiten bei der Steuerung des Heilverfahrens kommen. Eine fortwährende Überprüfung des Behandlungsverlaufs und der Indikation ist daher in jedem Fall unverzichtbar. Außerdem muss vor der Empfehlung zum Einsatz von niederenergeti- schem, gepulstem Ultraschall eine überschlagsmäßige Kosten-Nutzen-Analyse erfolgen [13]. Im Fall von frischen, unkomplizierten Frakturen halten wir den Einsatz des Verfahrens trotz nachgewiesener Verkürzung der Heilungszeit $[6,11]$ im Regelfall für nicht gerechtfertigt, da bei knapper werdenden Ressourcen der Kostenträger im Gesundheitswesen ökonomische Gesichtspunkte dagegen sprechen.

\section{Korrespondierender Autor \\ Dr.S.A. Esenwein}

Chirurgische Klinik mit Poliklinik, BG Kliniken Bergmannsheil, Ruhr-Universität Bochum,

Bürkle-de-la-Camp-Platz 1, 44789 Bochum,

E-Mail: Stefan.A.Esenwein@ruhr-uni-bochum.de

\section{Literatur}

1. Buckley MJ, Banes AJ, Levin LG, Sumpio BE, Sato M, Jordan R, Gilbert J, Link GW, Tan Son Tay R (1988) Osteoblasts increase their rate of division and align in response to cyclic, mechanical tension in vitro. Bone Miner 4: 225-236

2. Cook SD, Ryaby JP, McCabe J, Frey JJ, Heckman JD (1997) Acceleration of tibia and distal radius fracture healing in patients who smoke. Clin Orthop 337: 198-207

3. Duarte $L R$ (1983) The stimulation of bone growth by ultrasound. Arch Orthop Trauma Surg 101: 153-159

4. Esenwein SA, Hopf F, Pommer A, Kutscha-Lissberg F, Kollig E, Muhr G (2001) Die Anwendung von niederenergetischem, gepulstem Ultraschall im Falle des drohenden Regeneratversagens bei der Kallusdistraktion klinische Ergebnisse. Hefte Unfallchirurg 283: 236-237

5. Esenwein SA, Hering S, Muhr G, Hopf F, Kutscha-Lissberg F, Köller M (2003) Einfluss von niederenergetischem, gepulstem Ultraschall auf osteoblastäre Zelllinien unterschiedlicher Herkunft in vitro. Publikation in Vorbereitung

6. Heckman JD, Ryaby JP, McCabe J, Frey JJ, Kilcoyne RF (1994) Acceleration of tibial fracture-healing by noninvasive, low-intensity pulsed ultrasound.J Bone Joint Surg 76-A: 26-34

7. Klug W (1983) Stimulierung der Knochenbruchheilung durch Ultraschall. Tierexperimentelle Studie. Beitr Orthop Traumatol 30: 670-680

8. Klug W, Knoch HG (1986) Durch biophysikalische Untersuchungen Quantifizierung der Knochenbruchheilung nach Ultraschallstimulation von distalen Radiusfrakturen. Beitr Orthop Traumatol 33: 384-391

9. Knoch HG (1967) Der Einfluss von Nieder- und Hochfrequenzschwingungen auf das Kallusgewebe im Tierexperiment.Zentralbl Chir 92: 1784-1799

10. Knoch HG (1990) Knochenbruchheilung mit Ultraschall. Springer, Berlin Heidelberg New York

11. Kristiansen TK, Ryaby JP, McCabe J, Frey JJ, Roe LR (1997) Accelerated healing of distal radial fractures with the use of specific, low-intensity ultrasound. A multicenter, prospective, randomized, double-blind, placebo-controlled study.J Bone Joint Surg 79-A: 961-973

12. Machen MS, Tis JE, Inoue N, Meffert RH, Chao EY, McHale KA (2002) The effect of low intensity pulsed ultrasound on regenerate bone in a less-than-rigid biomechanical environment.J Biomed Mater Res 12: 239-247
13. Mayr E (2001) Niedrigintensive Ultraschallbehandlung bei Frakturheilungsproblemen. Trauma Berufskrankh [Suppl 2] 3: 276-279

14. Mayr E, Wagner S, Ecker M, Rüter A (1999) Die Ultraschalltherapie bei Pseudarthrosen - 3 Fallbeschreibungen. Unfallchirurg 102: 191-196

15. Mayr E, Rudzki M, Borchard B, Rüter A (2000) Beschleunigt niedrig intensiver, gepulster Ultraschall die Heilung von Skaphoidfrakturen? Handchir Mikrochir Plast Chir 32: 115-122

16. Mayr E, Laule A, Suger G, Rüter A, Claes L (2001) Radiographic results of callus distraction aided by pulsed low-intensity ultrasound.J Orthop Trauma 15: 407-414

17. Mayr E, Möckl C, Lenich A, Ecker M, Rüter A (2002) Ist niedrig-intensiver Ultraschall bei der Behandlung von Frakturheilungsstörungen wirksam? Unfallchirurg 105: 108-115

18. Miyazono K, Kusanagi K, Inoue H (2001) Divergence and convergence of TGF-beta/BMP signaling.J Cell Physiol 187: 265-276

19. Muhr G (1998) Pseudarthrosentherapie mit ExogenUltraschall. Rundschreiben des Landesverbandes Rheinland-Westfalen der gewerblichen Berufsgenossenschaften an die Unfallversicherungsträger Nr. 14/98 vom 1.9.1998. Landesverband Rheinland-Westfalen der gewerblichen Berufsgenossenschaften, Düsseldorf

20. Naruse K, Mikuni-Takagaki Y, Azuma Y, Ito M, Oota T, Kameyama K, Itoman M (2000) Anabolic response of mouse bone-marrow-derived stromal cell clone ST2 cells to low-intensity pulsed ultrasound. Biochem Biophys Res Commun 268: 216-220

21. Nolte PA, Klein-Nulend J, Albers GH, Marti RK, Semeins CM, Goei SW, Burger EH (2001) Low-intensity ultrasound stimulates endochondral ossification in vitro. J Orthop Res 19: 301-307

22. Parvizi J, Wu CC, Lewallen DG Greenleaf JF, Bolander ME (1999) Low-intensity ultrasound stimulates proteoglycan synthesis in rat chondrocytes by increasing aggrecan gene expression. J Orthop Res 17: 488-494

23. Pommer A, Muhr G (2001) Gepulster Ultraschall. Trauma Berufskrankh [Suppl 1] 3: 76-79

24. Sato W, Matsushita T, Nakamura K (1999) Acceleration of increase in bone mineral content by low-intensity ultrasound energy in leg lengthening. J Ultrasound Med 18: 699-702

25. Schmelz A (2001) Effect of in vitro low intensity ultrasound treatment in fibroblast and osteoblast proliferation. Abstract Book der $1^{\text {st }}$ SICOT/SIROT Annual International Conference und XII.SICOT Trainees' Meeting in Paris/Frankreich vom 30.8.2001-1.9.2001, Abstract 50: 10

26. Sun JS, Hong RC, Chang WH, Chen LT, Lin FH, Liu HC (2001) In vitro effects of low-intensity ultrasound stimulation on the bone cells.J Biomed Mater Res 57: 449-456

27. Tis JE, Meffert RH, Inoue N, McCarthy EF, Machen MS, McHale KA, Chao EY (2002) The effect of low intensity pulsed ultrasound applied to rabbit tibiae during the consolidation phase of distraction osteogenesis. J Orthop Res 20: 793-800

28. Warden SJ, Favaloro JM, Bennell KL, McMeeken JM, Ng KW, Zajac JD, Wark JD (2001) Low-intensity pulsed ultrasound stimulates a bone-forming response in UMR-106 cells. Biochem Biophys Res Commun 286: $443-450$

29. Wu CC, Lewallen DG, Bolander ME, Bronk J, Kinnick RR, Greenleaf JF (1996) Exposure to low intensity ultrasound stimulates aggrecan gene expression by cultured chondrocytes. Trans Orthop Res Soc 21: 622

30. Yang KH, Parvizi J, Wang SJ, Lewallen DG, Kinnick RR Greenleaf JF, Bolander ME (1996) Exposure to lowintensity ultrasound increases aggrecan gene expression in a rat femur fracture model. J Orthop Res 14: 802-809 